\title{
A Novel Sliding Mode Control Algorithm for an Active Suspension System Considering with the Hydraulic Actuator
}

\author{
Duc Ngoc Nguyen $^{a}$ (D), Tuan Anh Nguyen ${ }^{\text {* }}$ (D), Ngoc Duyen Danga ${ }^{\text {(D) }}$
}

aAutomotive Engineering Department, Thuyloi University, 175 Tay Son, Dong Da, Hanoi, Viet Nam. Email: anhngtu@tlu.edu.vn; ndn@tlu.edu.vn; duyen.dndp@tlu.edu.vn

* Corresponding author

https://doi.org/10.1590/1679-78256883

\begin{abstract}
The suspension system has the role of regulating and extinguishing oscillations in the vehicle. To improve stability and comfort, the active suspension system is proposed to replace the passive suspension system. There are many algorithms used for active suspension system control, such as PID, LQR, Fuzzy, etc. Among them, the nonlinear control method which uses the SMC algorithm gives a stable performance. This research proposes the use of the SMC algorithm to control the operation of the active suspension system equipped with a quarter dynamics model. The process of linearization of the hydraulic actuator is presented in the paper. As a result of the simulation, the values of displacement and acceleration of the sprung mass were significantly reduced when the vehicle used the active suspension system controlled by the SMC algorithm. The SMC controller established in this paper provides stability in many situations. Therefore, the vehicle's smoothness and comfort have been significantly improved. In the future, intelligent algorithms can be combined with SMC algorithms to improve the efficiency of the controller.
\end{abstract}

\section{Keywords}

Vehicle dynamics, Sliding Mode Control (SMC), Nonlinear Control Method, Active Suspension System, Hydraulic Actuator.

\section{Graphical abstract}
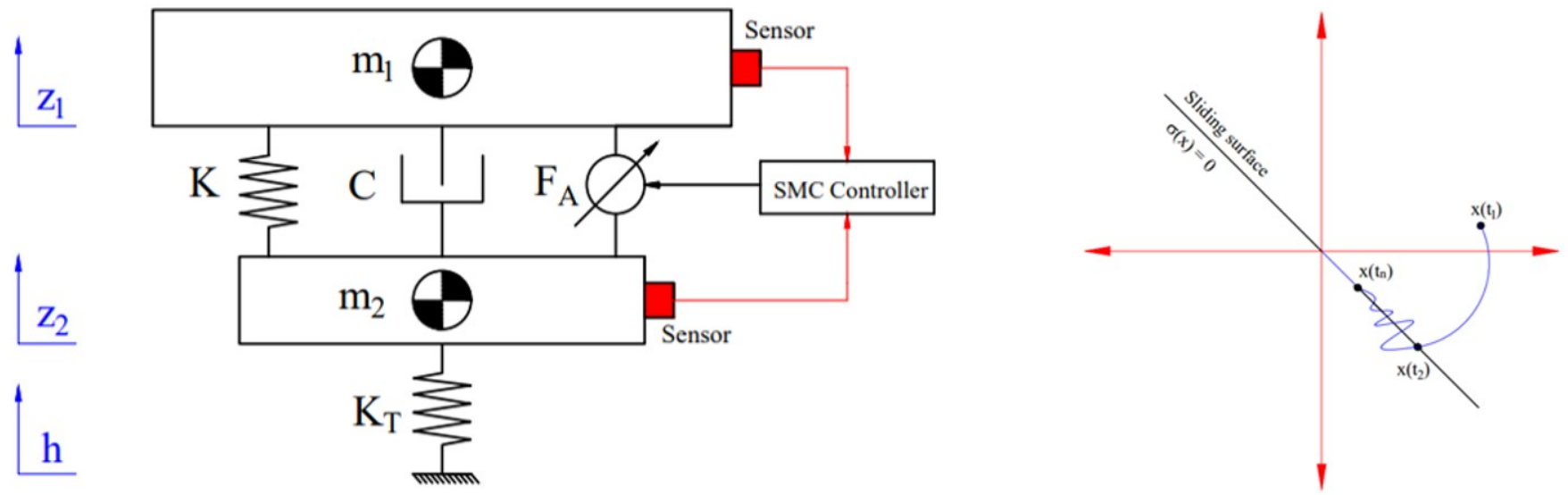


\section{INTRODUCTION}

Vehicle oscillation has a great influence on the health of passengers and the quality of goods. There are many causes of vehicle oscillations, in which the excitation from the road surface is the direct cause of these oscillations. To evaluate the oscillation, several parameters are considered, such as displacement of the sprung mass, acceleration of the sprung mass, roll angle, etc. These parameters are evaluated based on the maximum value and the average value which is calculated according to the Root Mean Square (RMS) (Tian and Nguyen, 2020). In addition, the oscillation frequency should also be considered.

$$
R M S=\sqrt{\frac{1}{T} \int_{0}^{T} f^{2}(\theta) d \theta}
$$

The suspension system on the vehicle has the role of regulating and extinguishing the oscillations of the vehicle. The conventional passive (mechanical) suspension system consists of the spring, shock absorber, and lever arm (Nguyen, 2021a). According to (Jugulkar et al., 2016; Jiregna and Sirata, 2020), the stiffness of the spring and damper is unchanged. Therefore, the smoothness of the vehicle will not be guaranteed. To improve the comfort of the vehicle, several solutions have been proposed. In (Abid et al., 2015), Abid et al. came up with a solution to use the air spring to replace the metal spring. According to Eskandary et al., the stiffness of the air spring can automatically change based on stimuli from the pavement (Eskandary et al., 2016). In addition, adjusting the stiffness of the damper also has a positive effect on the suspension system. The idea of using semi-active suspension was proposed by Aljarbouh and Fayaz (Aljarbouh and Fayaz, 2020). This suspension system uses the Electro-Rheological damper (ER), which is controlled by an electric current. When current is supplied, a magnetic field will appear inside the damper. This causes the extremely small iron particles to arrange tightly. Since then, the damping's stiffness has changed (Xiao et al., 2018). Besides changing the stiffness of the spring and damper, the idea of integrating a hydraulic actuator into the suspension was also proposed. According to (Wang et al., 2018), this suspension system is called the active suspension system.

There are many methods used to control the active suspension system. In (Anh, 2020), Anh used the PID algorithm with three coefficients $K_{P}, K_{1}$, and $K_{D}$ to reduce the error of the system to the minimum value. In the case of the integral system being ideal, the coefficient KI can be neglected. Therefore, this controller is only for PD (Haddar et al., 2019). The parameters of the PID controller can be self-tuned through the self-learning algorithm. This is shown in the paper of Talib and Darus (Talib and Darus, 2013). In addition, these parameters can be optimized based on the PSO algorithm (Talib et al., 2021). According to Munawwarah and Yakub, the PID control algorithm can be combined with the Fuzzy control algorithm to improve the efficiency of the operation (Munawwarah and Yakub, 2021). Besides, the combination of the PID algorithm and ANN also brings high efficiency to the system (Heidari and Homaei, 2013). Recently, Nguyen has proposed double integrated active suspension system control that uses two hydraulic actuators by a PID controller (Nguyen, 2021b). According to this result, the values of displacement and acceleration of the sprung mass have been significantly reduced. In fact, the PID controller is cheap and durable. However, this controller is only suitable for SISO systems. For systems with multiple inputs and multiple outputs (MIMO), the use of an LQR controller is a reasonable solution (Maurya and Bhangal, 2018). The LQR control algorithm helps to optimize the cost function. This is demonstrated by Nagarkar et al. (Nagarkar et al., 2011). To use this algorithm, the equations describing the oscillation state of the vehicle must be reduced to the state matrix (Rodriguez-Guevara et al., 2021). The unknown matrices of the system can be determined through the Riccati algebraic equation (Xia et al., 2015). In (Pang et al., 2017), Pang et al. proposed the use of a Gaussian filter for the LQR controller. Therefore, this controller is given a new name, and it is LQG. The signal of the LQG controller is more stable than that of the LQR controller because of the Gaussian filter that is fitted (Chen et al., 2018; Gomonwattanapanich et al., 2020). Besides, several other linear control algorithms have also been used for active suspension systems (Bello et al., 2015; Chai and Sun, 2010).

In some special cases, the linear control methods cannot meet the system's stability requirements. Therefore, several methods of nonlinear control and intelligent control have been proposed. The Robust control algorithm has been shown by Keleemullah et al. in their paper (Kaleemullah et al., 2019). The control parameter $\mathrm{H}_{\infty}$ is optimized based on Robust control algorithms (Rizvi et al., 2018). This controller provides a stable performance against changes from external stimuli (Singh et al., 2016; Gudarzi and Oveisi, 2014). Therefore, this algorithm can be applied to both electric vehicles that use the motor in the wheel (Wu et al., 2020). When external influences change continuously, Adaptive control is suitable for the active suspension system (Huang et al., 2018). According to Fu et al., the response of the Adaptive control algorithm is completely consistent with the change of pavement excitation (Fu et al., 2017). The response time of the Adaptive control algorithm is shorter than that of the LQR algorithm. According to Huang et al., the adaptation process 
is extremely gentle, and it occurs in a short period (Huang et al., 2015). This is also demonstrated by Sun and Gao in their study (Sun et al., 2013). To improve the efficiency of the Adaptive control algorithm, the Fuzzy algorithm has been combined. Therefore, the response of this controller was better guaranteed (Soleymani et al., 2012; Na et al., 2020). The input data of the Fuzzy controller will have to go through the fuzzification process (Palanisamy and Karuppan, 2016). According to Mrazgua, the output signal of the controller is determined based on the fuzzy rule, which has been established previously (Mrazgua et al., 2021). Besides, the neural control algorithms also have positive effects on the active suspension system (Liu et al., 2019; Aela et al., 2020).

The Sliding Mode Control algorithm can be used for complex oscillating nonlinear systems. In (Nguyen, 2021c), Nguyen used the Sliding Mode Control algorithm (SMC) for the quarter dynamics model. This oscillating system is an unstable nonlinear form. According to Sam and Osman, hydraulic actuators have been linearized (Sam and Osman, 2006). It becomes a function of the in put voltage signal. In (Bai and Guo, 2018), Bai and Guo introduced the Sliding Mode Control algorithm with five state variables. However, the error signal is only derivative three times. Therefore, the stability of the control process is still not guaranteed. In (Wei and Su, 2020), Wei and Su introduced a way to select the sliding surface for the SMC algorithm. Besides, some parameters of this controller are also optimized based on the PSO algorithm. In addition, the Sliding Mode Control algorithm can also be combined with other linear control and intelligent control methods (Sam et al., 2007; Li et al., 2019; Lin et al., 2019; Zhao et al., 2015; Alves et al., 2014; Sun and Zhao, 2020).

This research aims to establish a nonlinear control algorithm for the active suspension system. The SMC algorithm was chosen for this purpose. Different from previous studies, this paper details the linearization of hydraulic actuators. Besides, the process of designing the controller with the state variables of the oscillator model is also clearly shown. This linearization process is completely novel and unique. The results of the paper were obtained from the simulation, which was carried out in the Matlab-Simulink environment. The specific content of the paper is presented below.

\section{MATERIAL AND METHOD}

\subsection{Quarter dynamics model}

With the quarter dynamics model (Figure 1), the equations describing the vehicle's oscillation are given as follows:

$$
\begin{aligned}
& m_{1} \ddot{z}_{1}=F_{K}+F_{C}+F_{A} \\
& m_{2} \ddot{z}_{2}=F_{K T}-F_{K}-F_{C}-F_{A}
\end{aligned}
$$

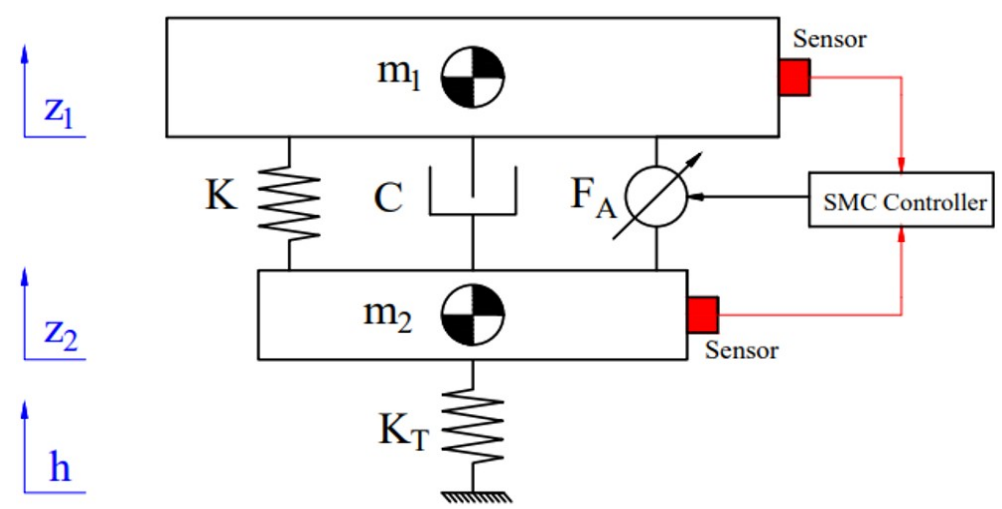

Figure 1 Quarter dynamics model.

Where:

Force of the spring:

$F_{K}=K\left(z_{2}-z_{1}\right)$

Force of the damper:

$F_{C}=C\left(\dot{z}_{2}-\dot{z}_{1}\right)$ 
Force of the tire:

$F_{K T}=K_{T}\left(h-z_{2}\right)$

The above equations are rewritten as:

$m_{1} \ddot{z}_{1}=-K z_{1}-C \dot{z}_{1}+K z_{2}+C \dot{z}_{2}+F_{A}$

$m_{2} \ddot{z}_{2}=K z_{1}+C \dot{z}_{1}-\left(K+K_{T}\right) z_{2}-C \dot{z}_{2}-F_{A}+K_{T} h$

\subsection{Hydraulic actuator dynamics model}

The structure of the hydraulic actuator is shown in Figure 2. This is a complex hydraulic piston. The displacement of the piston depends on the displacement of the servo valves.

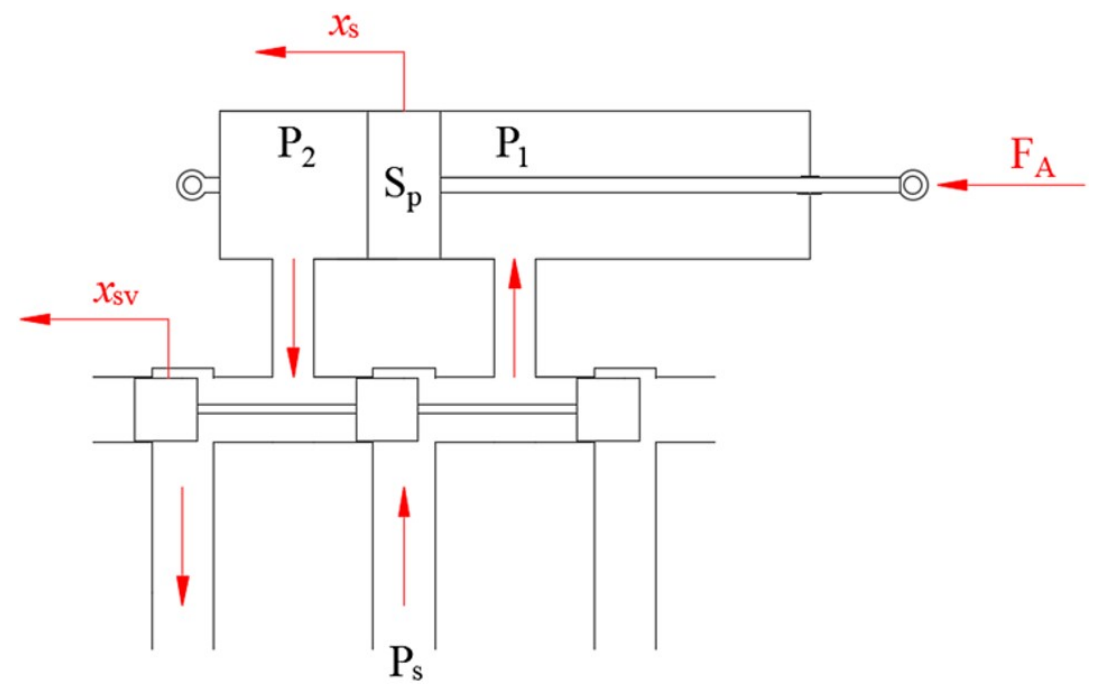

Figure 2 Hydraulic actuator.

The force generated by the hydraulic actuator is proportional to the pressure change between the two chambers $\Delta P:$

$F_{A}=S_{p} \Delta P$

The displacement of servo valve $x_{s v}$ is controlled by the current signal $i(t)$ :

$x_{s v}=\frac{1}{t} \int\left(k_{s v} i(t)-x_{s v}\right) d t$

The flow of liquid inside the cylinder is calculated according to equation (11):

$Q=\frac{s_{3}}{s_{1}} x_{s v} \sqrt{P_{s}-\operatorname{sgn}\left(x_{s v}\right) \Delta P}$

The pressure difference between the two chambers of the cylinder is a complex function:

$\Delta P=\sigma_{1} \int\left(Q-\frac{\sigma_{2}}{\sigma_{1}} \Delta P-S_{p} \dot{x}_{s}\right) d t$ 
From (11) and (12), the change in pressure $\triangle P$ can be rewritten as:

$\Delta P=\sigma_{3} \int\left(x_{s v} \sqrt{P_{s}-\operatorname{sgn}\left(x_{s v}\right) \Delta P}\right) d t-\sigma_{2} \int \Delta P d t-\sigma_{1} S_{p} \int \dot{x}_{s} d t$

Combining (9) and (13), the impact force that is generated by the hydraulic actuator can be calculated as equation (14):

$F_{A}=S_{P} \Delta P=S_{P}\left(\sigma_{3} \int\left(x_{s v} \sqrt{P_{s}-\operatorname{sgn}\left(x_{s v}\right) \Delta P}\right) d t-\sigma_{2} \int \Delta P d t-\sigma_{1} S_{p} \int \dot{x}_{s} d t\right)$

\subsection{Sliding theory}

Consider the SISO nonlinear object of order $n$, which is described by the following equations of state:

$\left\{\begin{array}{l}\dot{x}=f(x)+g(x) u \\ y=h(x)\end{array}\right.$

Where:

$x=\left[\begin{array}{llll}x_{1} & x_{2} & \ldots & x_{n}\end{array}\right]^{T} \in R^{n}$ is the state vector of the system

$u \in R^{n}$ is the input signal

$y \in R^{n}$ is the output signal

$f(x) \in R^{n}, g(x) \in R^{n}$ are smooth function vectors describing the kinematics of the system

$h(x) \in R^{n}$ is a smooth function describing the relationship between the state variable and the output

As required, the output signal $y(t)$ is controlled according to the set signal $y_{s}(t)$. The object's input and output relationship can be represented as follows:

$y^{(n)}=a(x)+b(x) u$

Where:

$a(x)=L_{f}^{(n)} h(x)$

$b(x)=L_{g} L_{f}^{(n-1)} h(x)$

With:

$L_{f} h(x)=\frac{\partial h(x)}{\partial x} f(x)=\left[\begin{array}{llll}\frac{\partial h(x)}{\partial x_{1}} & \frac{\partial h(x)}{\partial x_{2}} & \ldots & \frac{\partial h(x)}{\partial x_{n}}\end{array}\right]\left[\begin{array}{c}f_{1}(x) \\ f_{2}(x) \\ \ldots \\ f_{n}(x)\end{array}\right]$

$L_{g} L_{f}^{(k)} h(x)=\frac{\partial\left(\frac{\partial L_{f}^{(k-1)} h(x)}{\partial(x)} f(x)\right)}{\partial(x)} g(x)$ 
In order for the point to move to the desired position, the trajectory of the motion of the point must be determined. This trajectory is called the slip surface $\sigma(x)$ (Figure 3).

$\sigma(x)=e^{(n-1)}+k_{1} e^{(n-2)}+\ldots+k_{n-2} \dot{e}+k_{n-1} e$

Where:

$e(t)$ is the error of the system

$e(t)=y_{s}(t)-y(t)$

$k_{i}$ is the coefficient of the Hurwitz polynomial

$P(s)=s^{n-1}+k_{1} s^{n-2}+\ldots+k_{n-2} s+k_{n-1}$

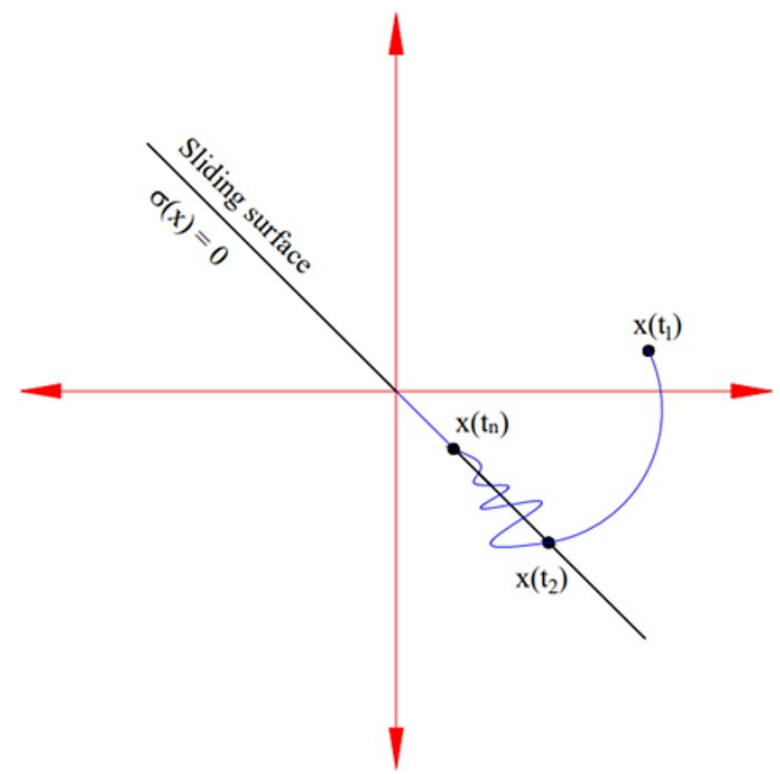

Figure 3 Sliding surface.

According to Lyapunov's stability theory:

$V=\frac{1}{2} \sigma^{2}$

Take the derivatives of both sides of (24):

$\dot{V}=\sigma \dot{\sigma}$

Because $\sigma \rightarrow 0 \Leftrightarrow \dot{V}<0$

Take the derivatives of both sides of (21):

$\dot{\sigma}=e^{(n)}+k_{1} e^{(n-1)}+\ldots+k_{n-2} \ddot{e}+k_{n-1} \dot{e}=y_{s}^{(n)}-y^{(n)}+k_{1} e^{(n-1)}+\ldots+k_{n-2} \ddot{e}+k_{n-1} \dot{e}$

$=y_{s}^{(n)}-a(x)-b(x) u+k_{1} e^{(n-1)}+\ldots+k_{n-2} \ddot{e}+k_{n-1} \dot{e}=-K \operatorname{sgn}(\sigma)$

Therefore, the control signal $u(t)$ can be defined as: 
$u(t)=\frac{1}{b(x)}\left[-a(x)+y_{s}^{(n)}+k_{1} e^{(n-1)}+k_{2} e^{(n-2)}+\ldots+k_{n-2} \ddot{e}+k_{n-1} \dot{e}+K \operatorname{sgn}(\sigma)\right]$

\subsection{Establishing the Sliding Mode Control algorithm}

The control system diagram is shown in Figure 4. The actuator of the active suspension system is a hydraulic piston.

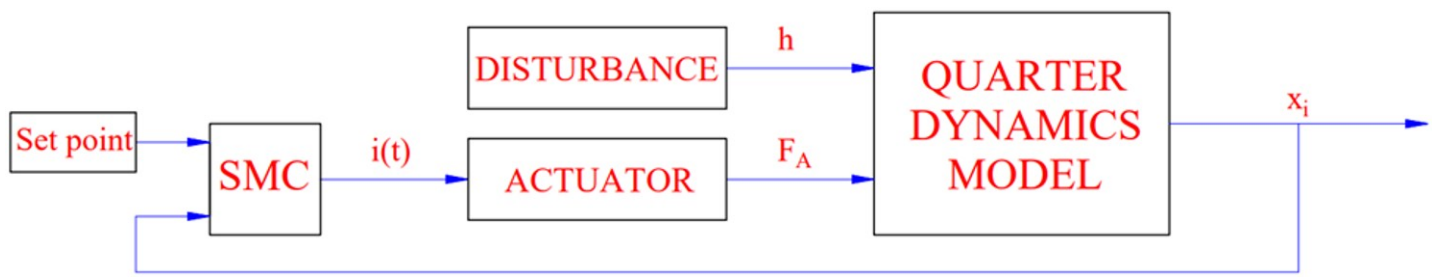

Figure 4 Control schematic.

According (10):

$\tau \dot{x}_{s v}=k_{s v} i(t)-x_{s v} \Leftrightarrow x_{s v}=k_{s v} i(t)-\tau \dot{x}_{s v}$

Because:

$\left\{\begin{array}{l}\left|k_{s v}\right| \approx|\tau| \\ \left|\dot{x}_{s v}\right| \ll \tau\end{array} \Rightarrow x_{s v} \approx k_{s v} i(t)\right.$

Take the derivatives of both sides of (14):

$\dot{F}_{A}=S_{p}\left(\sigma_{s} x_{s v} \sqrt{P_{s}-\operatorname{sgn}\left(x_{s v}\right) \Delta P}-\frac{\sigma_{2}}{S_{p}} F_{A}-\sigma_{1} S_{p} \dot{x}_{s}\right)$

Because: $\operatorname{sgn}\left(x_{s v}\right) \Delta P \ll P_{s}$

$\Rightarrow \dot{F}_{A} \approx \underbrace{S_{p} \sigma_{3} \sqrt{P_{s}} k_{s v}}_{\gamma_{1}} i(t)-\underbrace{\sigma_{2}}_{\gamma_{2}} F_{A}-\underbrace{\sigma_{1} S_{p}^{2}}_{\gamma_{3}} \dot{x}_{s}$

The above equation can be rewritten in linear form as follows:

$\dot{F}_{A}=\gamma_{1} i(t)-\gamma_{2} F_{A}-\gamma_{3}\left(\dot{z}_{1}-\dot{z}_{2}\right)$

Taking (2) + (3), we get:

$m_{1} \ddot{z}_{1}+m_{2} \ddot{z}_{2}=K_{T} h-K_{T} z_{2}$

Equation (33) can be rewritten as:

$\ddot{z}_{1}=\frac{K_{T}}{\lambda m_{1}}\left(h-z_{2}\right)$

Let the state variables as follows:

$x_{1}=z_{1} \quad x_{2}=\dot{z}_{1} \quad x_{3}=z_{2} x_{4}=\dot{z}_{2} \quad x_{5}=F_{A}$ 
Taking the derivative of state variables:

$\dot{x}_{1}=x_{2} \quad \dot{x}_{2}=\frac{1}{m_{1}}\left(-K x_{1}-C x_{2}+K x_{3}+C x_{4}+x_{5}\right)$

$\dot{x}_{3}=x_{4} \quad \dot{x}_{4}=\frac{1}{m_{2}}\left(K x_{1}+C x_{2}-\left(K+K_{T}\right) x_{3}-C x_{4}-x_{5}\right)$

$\dot{x}_{5}=\gamma_{1} i(t)-\lambda_{3} x_{2}+\gamma_{3} x_{4}-\gamma_{2} x_{5}$

Assume that the output signal is the displacement of the sprung mass:

$y=z_{1}=x_{1}$

Take the derivatives of both sides of (35):

$\dot{y}=\dot{x}_{1}=x_{2}$

Taking the second derivative of (36):

$\ddot{y}=-\frac{K_{T}}{\lambda m_{1}} x_{3}$

Take the derivatives of both sides of (37):

$y^{(3)}=-\frac{K_{T}}{\lambda m_{1}} x_{4}$

The fourth derivative of the output signal has the following form:

$y^{(4)}=-\frac{K_{T}}{\lambda m_{1} m_{2}}\left(K x_{1}+C x_{2}-\left(K+K_{T}\right) x_{3}-C x_{4}-x_{5}\right)$

Take the derivatives of both sides of (39):

$y^{(5)}=\frac{K_{T}}{\lambda m_{1} m_{2}}\left[\begin{array}{l}K C\left(\frac{1}{m_{1}}+\frac{1}{m_{2}}\right) x_{1}+\left(\frac{C^{2}}{m_{1}}+\frac{C^{2}}{m_{2}}-K-\gamma_{3}\right) x_{2}-C\left(\frac{K}{m_{1}}+\frac{K+K_{T}}{m_{2}}\right) x_{3} \\ -\left(\frac{C^{2}}{m_{1}}+\frac{C^{2}}{m_{2}}-K-K_{T}-\gamma_{3}\right) x_{4}-\left(\frac{C}{m_{1}}+\frac{C}{m_{2}}+\gamma_{2}\right) x_{5}\end{array}\right]+\frac{K_{T} \gamma_{1}}{\lambda m_{1} m_{2}} i(t)$

$=\mu_{1} x_{1}+\mu_{2} x_{2}+\mu_{3} x_{3}+\mu_{4} x_{4}+\mu_{5} x_{5}+\frac{K_{T} \gamma_{1}}{\lambda m_{1} m_{2}} i(t)$

The error of the system:

$e(t)=y_{s}-y$

The sliding surface is designed based on the error signal $e(t)$ : 
$\sigma=e^{(4)}+k_{1} e^{(3)}+k_{2} \ddot{e}+k_{3} \dot{e}+k_{4} e$

The control signal is defined as (43):

$i(t)=\frac{\lambda \prod_{i=1}^{2} m_{i}}{K_{T} \gamma_{1}}\left[y_{s}^{(n)}-\sum_{j=1}^{n} \mu_{j} x_{j}+\sum_{s=1}^{n-1} k_{s} e^{(n-1-s)}+\operatorname{Psgn}\left(\sum_{m=0}^{n-1} \sum_{l=0}^{n-1} k_{m} e^{(n-1-l)}\right)\right]$

\section{RESULTS AND DISCUSSIONS}

\subsection{Simulation condition}

In this research, the excitation from the pavement has the form of a sine wave (Figure 5). The amplitude of the oscillation will be changed in two cases as shown in Table 1.

$h=A \sin (2 \pi f t+\varphi)$

Table 1 Parameters of the roughness on the road.

\begin{tabular}{cccc}
\hline & $\boldsymbol{A}(\mathbf{m m})$ & $\boldsymbol{f ( H z )}$ & $\boldsymbol{\varphi}(\mathbf{r a d})$ \\
\hline Case 1 & 50 & 3 & 0 \\
Case 2 & 100 & 3 & 0 \\
\hline
\end{tabular}

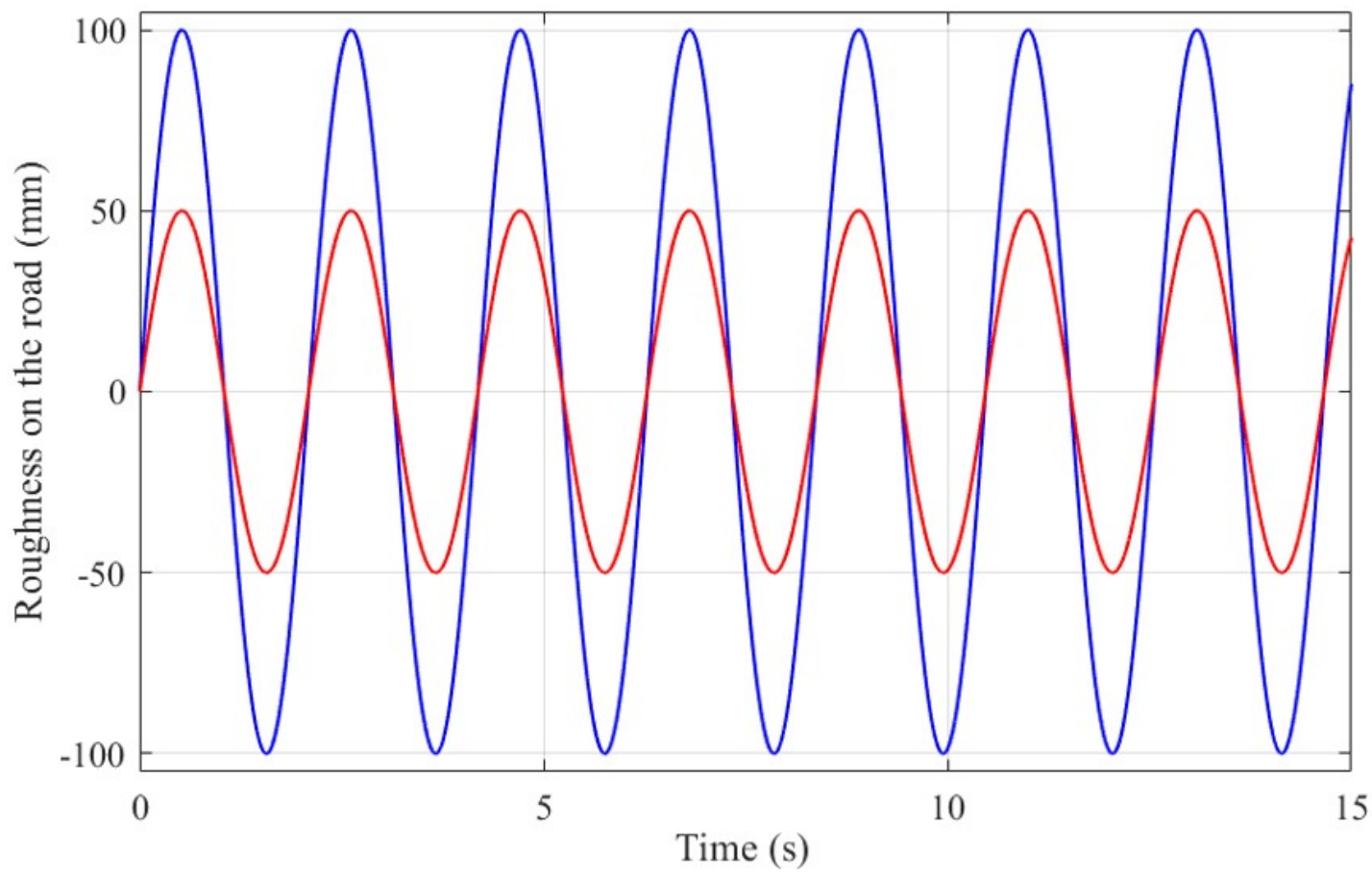

Figure 5 Roughness on the road.

The specifications of the reference vehicle and the hydraulic actuator are shown in Table 2 . 
Table 2 Specification of the vehicle and actuator.

\begin{tabular}{|c|c|c|c|}
\hline Description & Symbol & Value & Unit \\
\hline Sprung mass & $\mathrm{m}_{1}$ & 450 & $\mathrm{~kg}$ \\
\hline Unsprung mass & $\mathrm{m}_{2}$ & 45 & $\mathrm{~kg}$ \\
\hline Spring coefficient & K & $40 \times 10^{3}$ & $\mathrm{~N} / \mathrm{m}$ \\
\hline Tire coefficient & $\mathrm{K}_{\mathrm{T}}$ & $180 \times 10^{3}$ & $\mathrm{~N} / \mathrm{m}$ \\
\hline Damper coefficient & C & $3.5 \times 10^{3}$ & $\mathrm{Ns} / \mathrm{m}$ \\
\hline Actuator coefficient & $\sigma_{1}$ & $4.5 \times 10^{13}$ & $\mathrm{~N} / \mathrm{m}^{5}$ \\
\hline Actuator coefficient & $\sigma_{2}$ & 1 & $s^{-1}$ \\
\hline Actuator coefficient & $\sigma_{3}$ & $1.5 \times 10^{9}$ & $\mathrm{~N} / \mathrm{kg}^{1 / 2} \mathrm{~m}^{5 / 2}$ \\
\hline Time constant & $\tau$ & $2.5 \times 10^{-3}$ & s \\
\hline Piston cross-sectional area & $S_{p}$ & $3.5 \times 10^{-4}$ & $\mathrm{~m}^{2}$ \\
\hline Supply pressure & $\mathrm{P}_{\mathrm{s}}$ & 1056240 & $\mathrm{~N} / \mathrm{m}^{2}$ \\
\hline Servo valve gain & $\mathrm{k}_{\mathrm{sv}}$ & $1 \times 10^{-3}$ & $\mathrm{~m} / \mathrm{V}$ \\
\hline
\end{tabular}

\subsection{Simulation results}

Case 1:

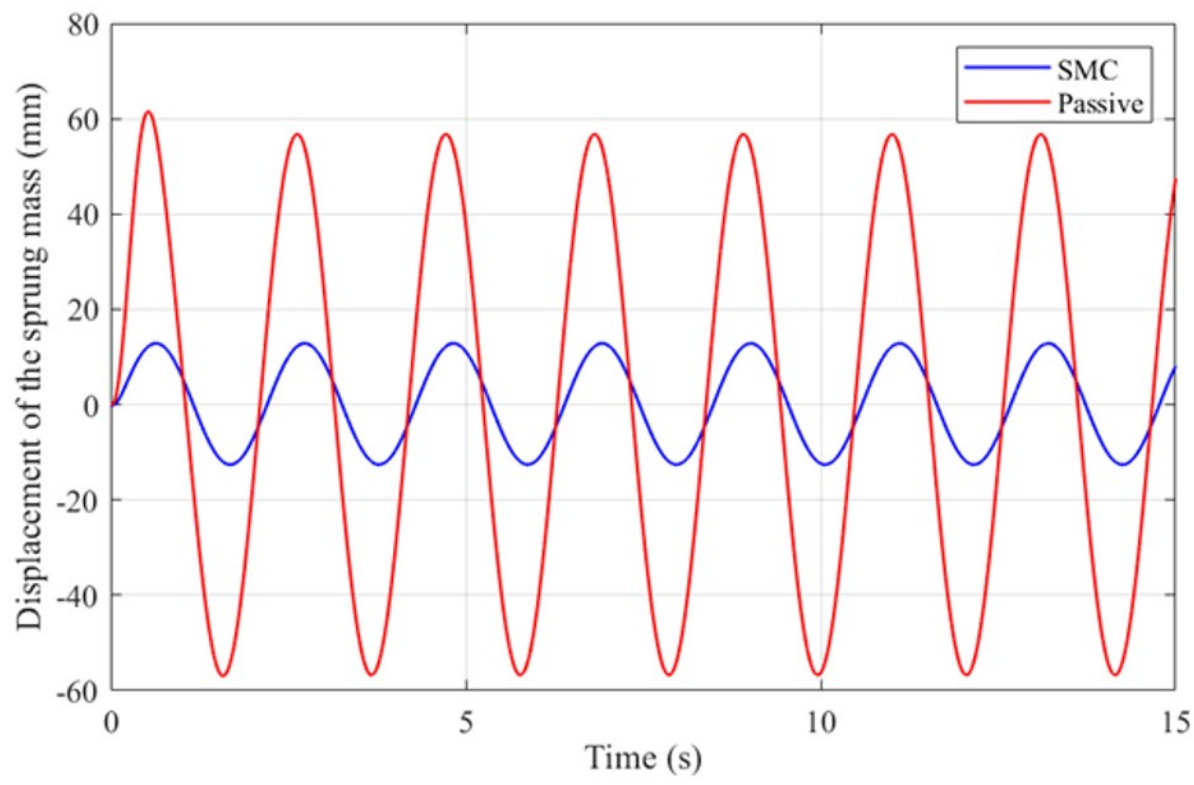

Figure 6 Displacement of the sprung mass (Case 1).

In this case, the amplitude of the excitation from the pavement will not be large. The change in displacement of the sprung mass is shown through the graph of Figure 6. According to this graph, if the vehicle only uses the conventional passive suspension system, the maximum value of the displacement of the sprung mass can reach 61.5 ( $\mathrm{mm}$ ) in the first phase. In subsequent phases, the peak value of the oscillation will decrease slightly, reaching 57.8 ( $\mathrm{mm}$ ). The average value of the oscillation which is calculated according to the RMS is $39.9(\mathrm{~mm})$. In contrast, if the vehicle uses the active suspension system, the displacement of the sprung mass will be significantly reduced. According to this result, its maximum displacement is only $12.9(\mathrm{~mm})$. Besides, the average value is also much lower, $\mathrm{RMS}=8.9(\mathrm{~mm})$.

The acceleration of the sprung mass is a measure of the vehicle's smoothness. If this value is too large, the smoothness of the vehicle will be affected. Therefore, it is necessary to determine the maximum and average value of the acceleration of the sprung mass. The change of acceleration with time is illustrated in Figure 7.

According to Figure 7, the maximum acceleration of the sprung mass is $1.295\left(\mathrm{~m} / \mathrm{s}^{2}\right)$ and $0.748\left(\mathrm{~m} / \mathrm{s}^{2}\right)$ respectively, corresponding to the two investigated situations. After this value is reached, the acceleration of the sprung mass will oscillate steadily with a smaller amplitude. The average value of the acceleration, which is calculated in terms of RMS, is $0.386\left(\mathrm{~m} / \mathrm{s}^{2}\right)$ and $0.086\left(\mathrm{~m} / \mathrm{s}^{2}\right)$, respectively. Obviously, if the vehicle uses only the conventional mechanical suspension system, the average value of acceleration is about 4.5 times that of the case where the suspension is controlled by the SMC nonlinear controller. 


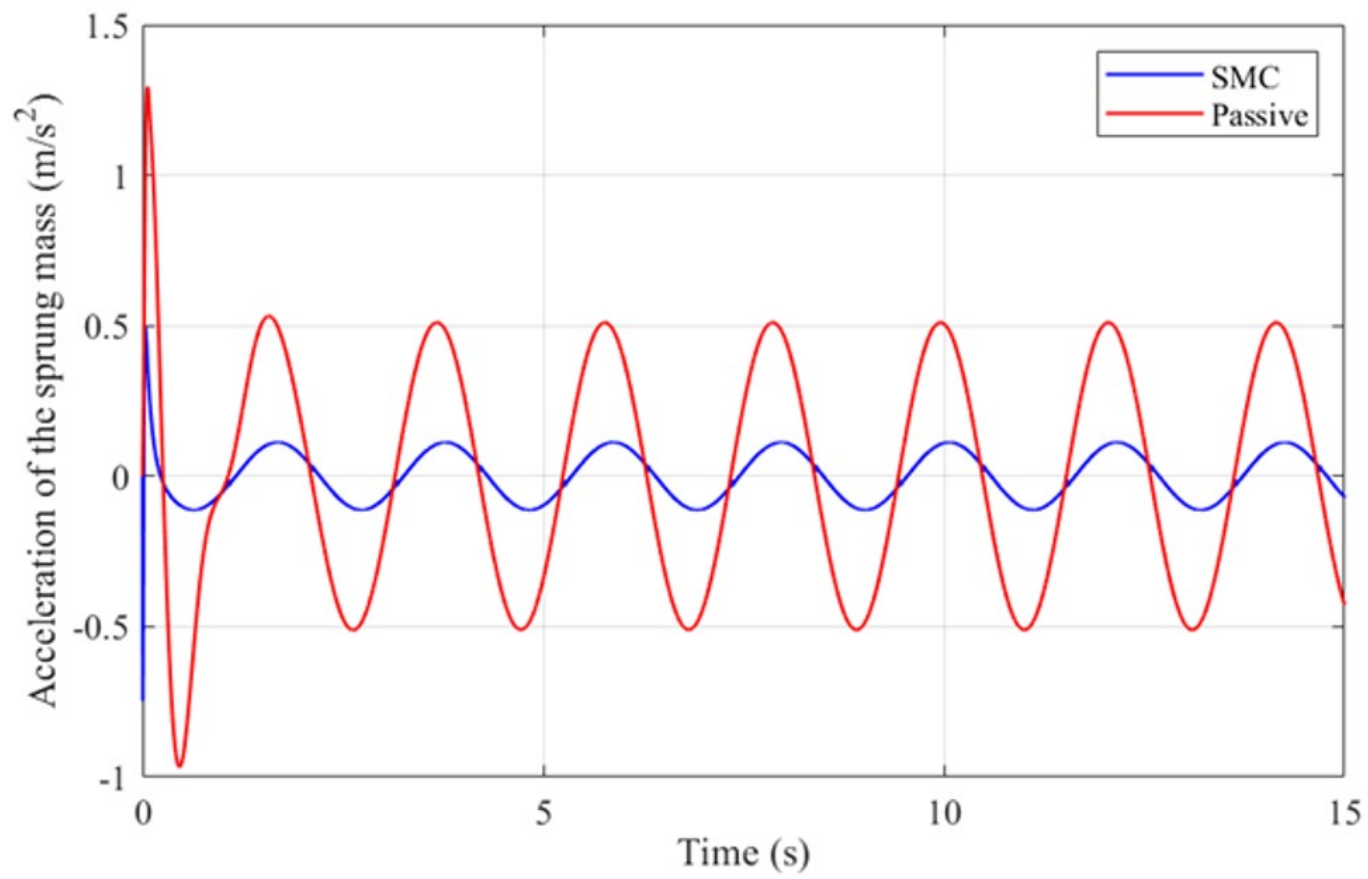

Figure 7 Acceleration of the sprung mass (Case 1).

According to some previous studies, the active suspension system will cause the unsprung mass to fluctuate more strongly than the passive suspension system. That causes the displacement and acceleration of the unsprung mass to be larger. Although this does not affect the smoothness of the vehicle too much. However, if the displacement and acceleration of the unsprung mass are guaranteed, vehicle comfort will be at its maximum.

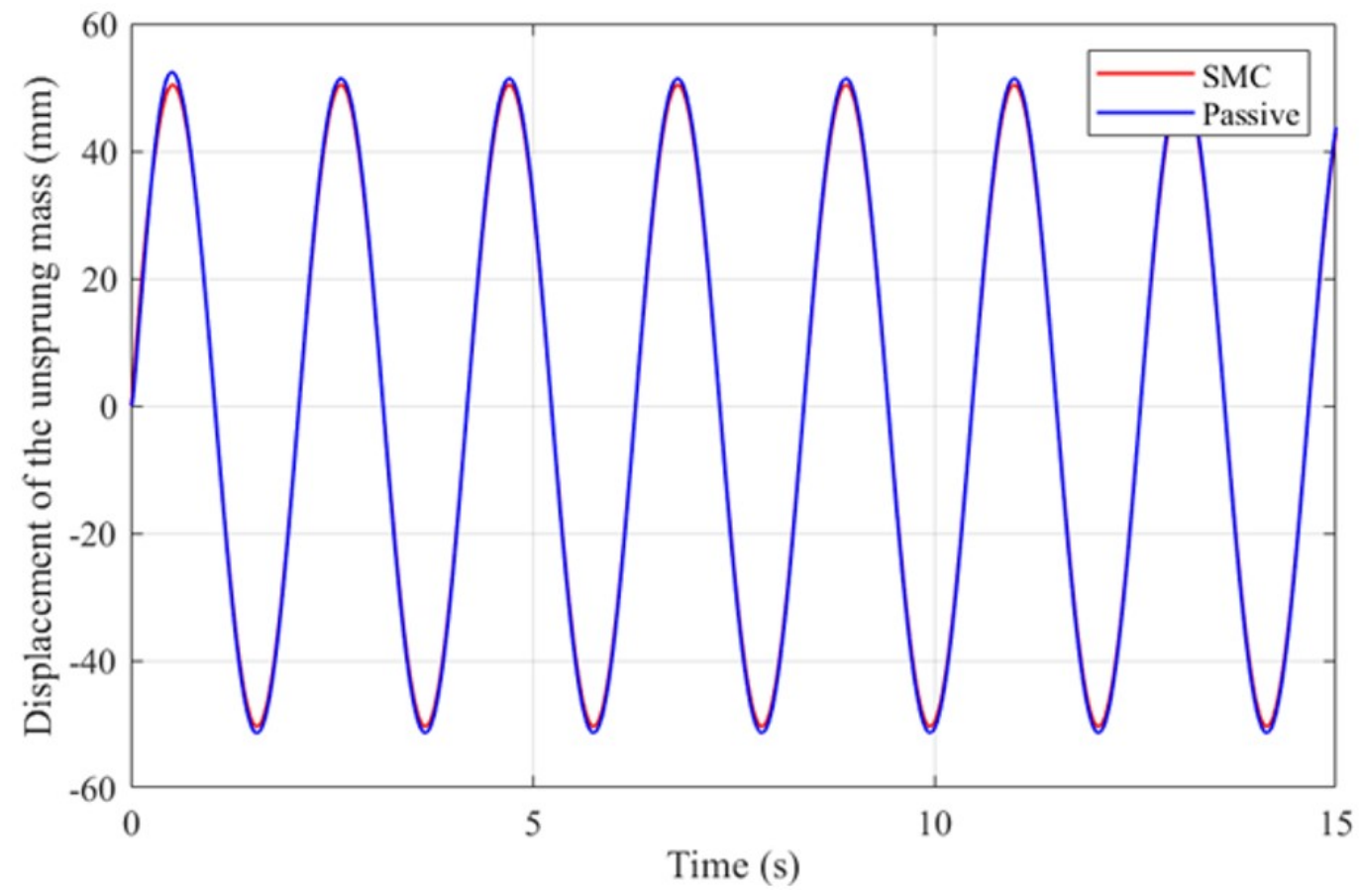

Figure 8 Displacement of the unsprung mass (Case 1).

When using the nonlinear controller, which is established in this paper, the displacement and acceleration of the unsprung mass are guaranteed to the smallest extent (Figure 8 and Figure 9). According to these graphs, the difference in amplitudes of the two cases is very small. Therefore, the smoothness and comfort of the vehicle are always guaranteed to be the best. 


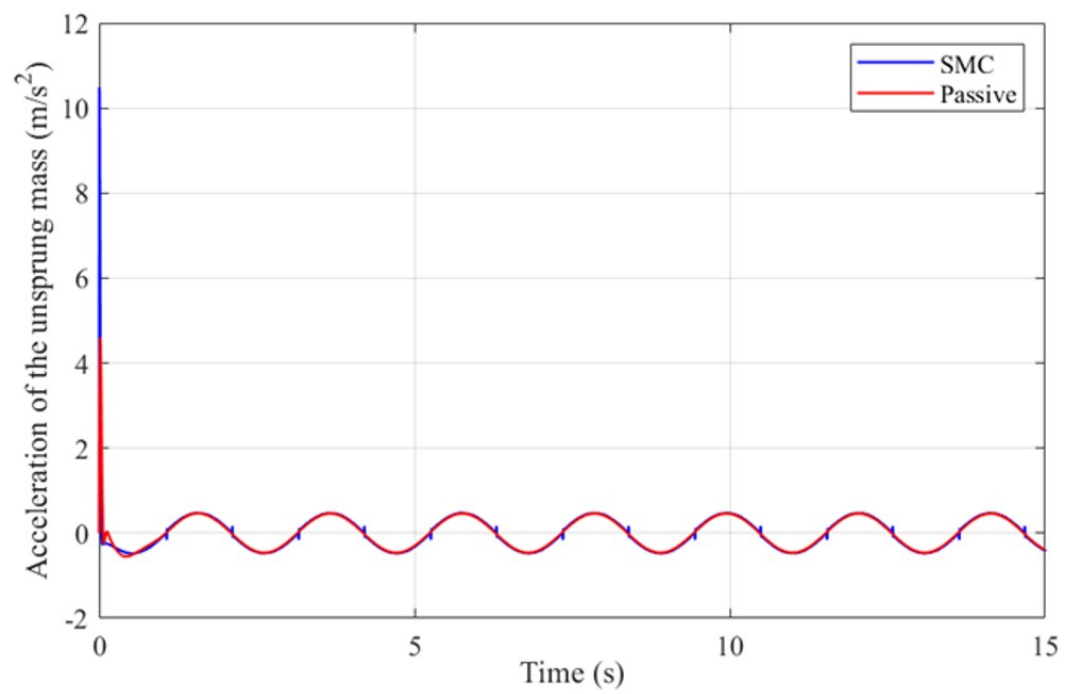

Figure 9 Acceleration of the unsprung mass (Case 1).

Case 2:

In the second case, the excitation from the pavement has a larger amplitude. Therefore, the oscillation of the vehicle will also be larger. Similar to the first case, the displacement of the sprung mass in the first phase will be the largest, reaching 123.1 (mm). In subsequent phases, this value is more stable, only about 115.3 (mm) (Figure 10). Meanwhile, the maximum value of displacement of the sprung mass when using the SMC controller is only 25.8 ( $\mathrm{mm}$ ). This value is only about $20.9 \%$ compared to the situation of vehicles using the passive suspension system.

In addition, the average value of the oscillation also has a significant difference corresponding to the two simulated situations. According to (1), their values are $79.8(\mathrm{~mm})$ and $17.8(\mathrm{~mm})$ respectively. As a result, the vehicle body will oscillate more stably and safely.

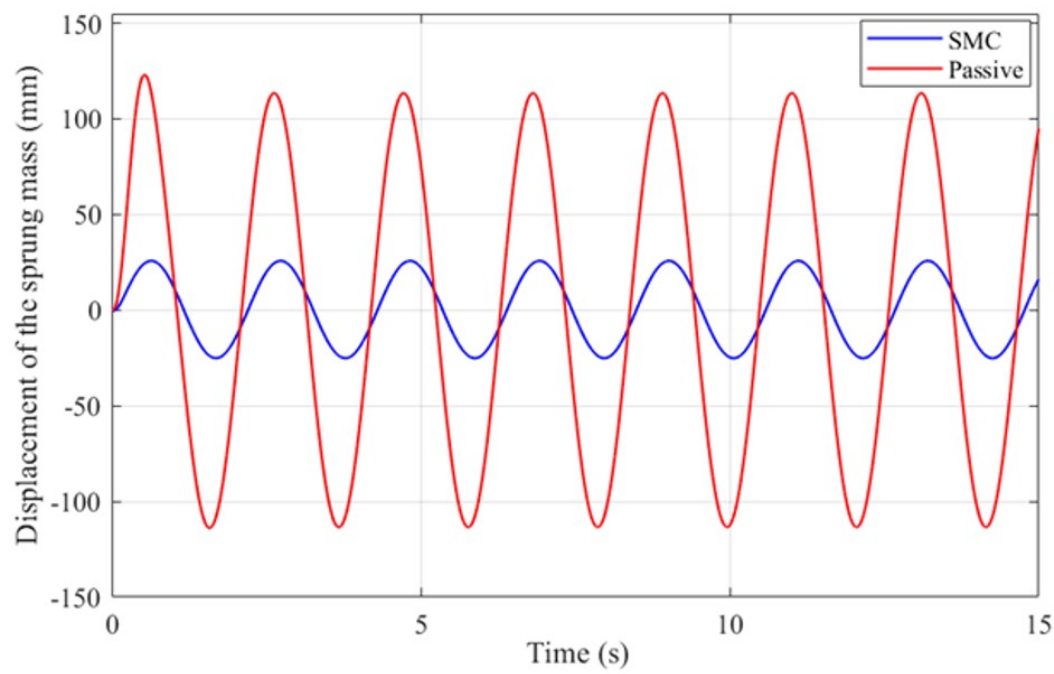

Figure 10 Displacement of the sprung mass (Case 2).

The change in acceleration of the sprung mass during the investigation period is shown in Figure 11. Accordingly, if the active suspension system is used to replace the conventional passive suspension system, the value of maximum acceleration will decrease from $2.590\left(\mathrm{~m} / \mathrm{s}^{2}\right)$ to $1.501\left(\mathrm{~m} / \mathrm{s}^{2}\right)$. After the end of the first phase, the oscillations in the later stages will gradually decrease and become more stable. The average value of acceleration in the case of vehicles using the mechanical suspension system is RMS $=0.773\left(\mathrm{~m} / \mathrm{s}^{2}\right)$. This value is 4.49 times higher than in the case of vehicles using the active suspension system controlled by the SMC algorithm, RMS $=0.172\left(\mathrm{~m} / \mathrm{s}^{2}\right)$. Obviously, the vehicle's stability and smoothness have been significantly improved when the vehicle is equipped with this advanced suspension system. 


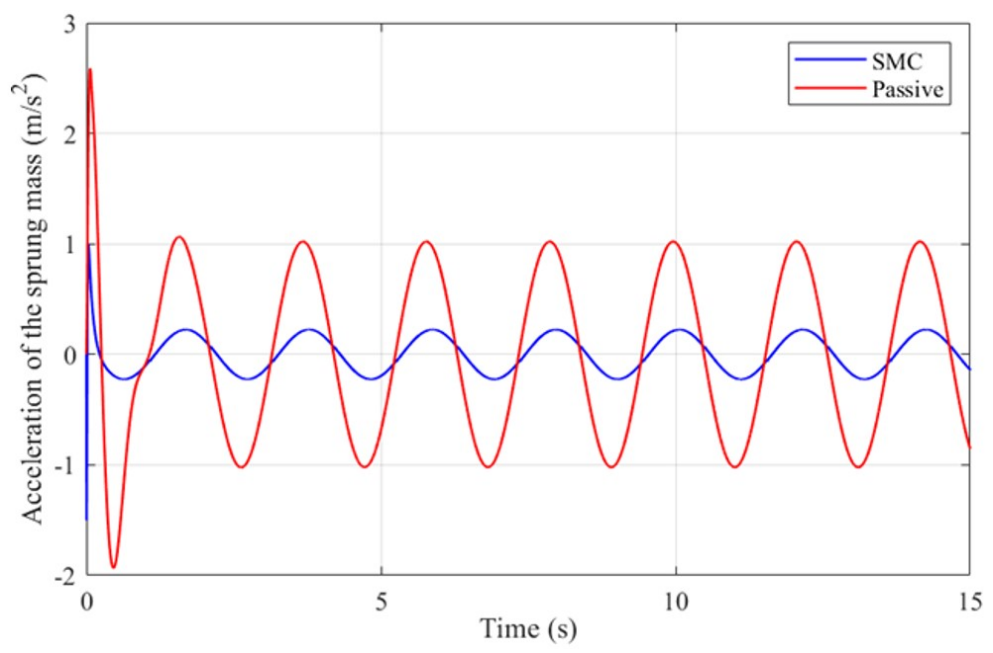

Figure 11 Acceleration of the sprung mass (Case 2).

The SMC nonlinear control algorithm helps to reduce the oscillation of the sprung mass. Besides, the oscillation of the unsprung mass is also guaranteed within certain limits. This is demonstrated by Figure 12 and Figure 13. Again, the oscillation of the whole vehicle is enhanced.

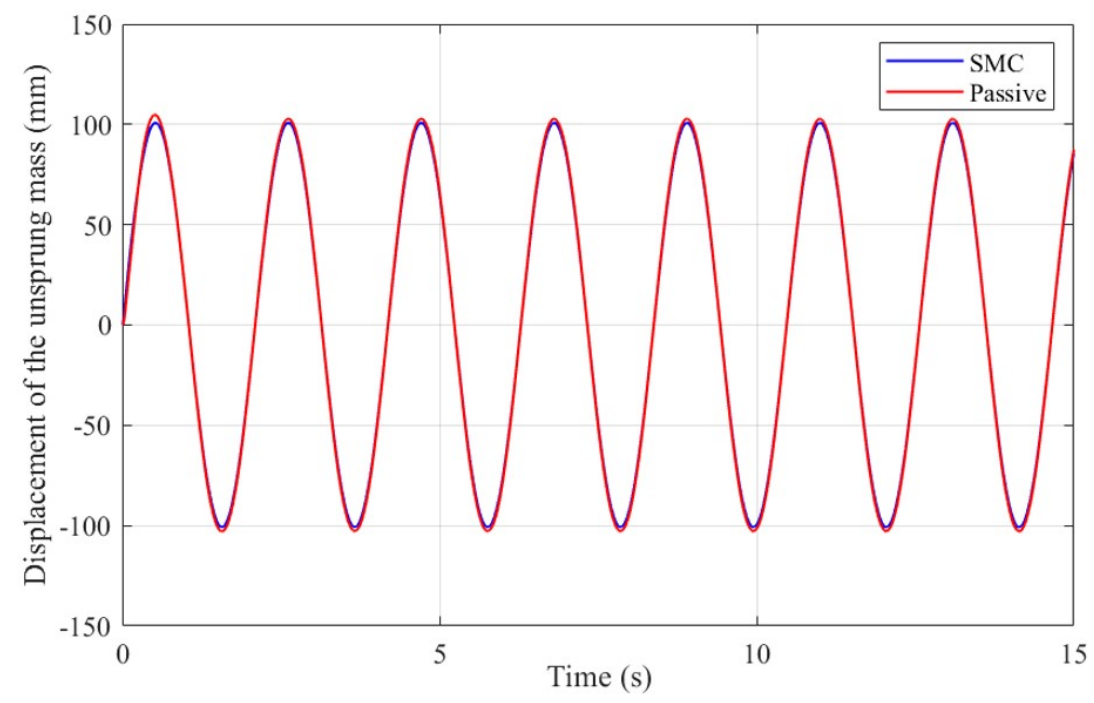

Figure 12 Displacement of the unsprung mass (Case 2).

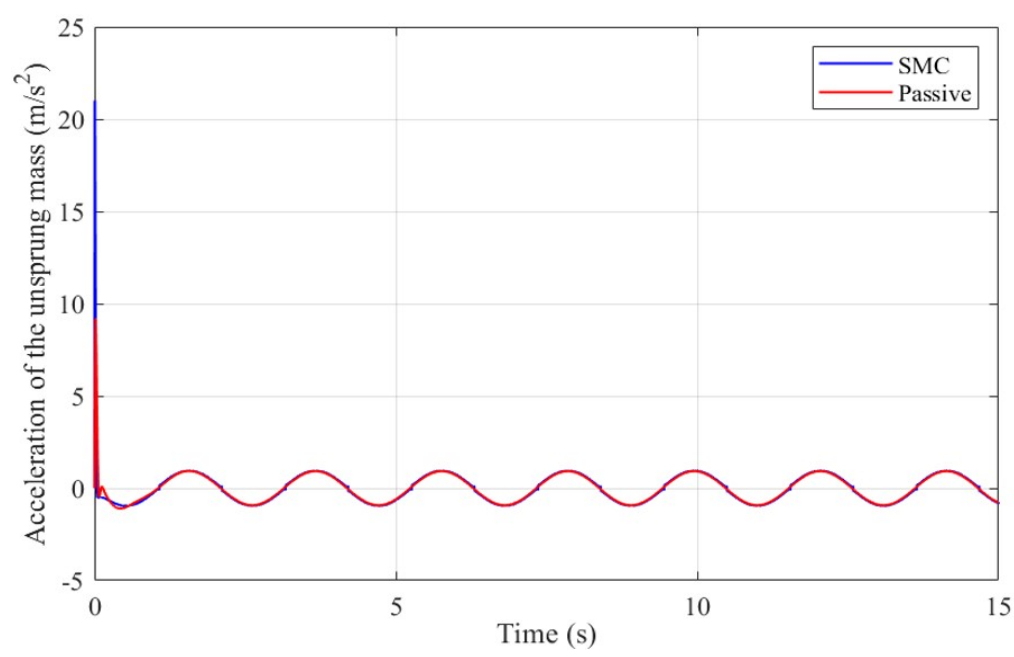

Figure 13 Acceleration of the unsprung mass (Case 2). 
The results of the simulation process are summarized as shown in Table 3 and Table 4.

Table 3 Results of the displacement of the sprung mass.

\begin{tabular}{ccccc}
\hline & \multicolumn{3}{c}{ Passive } & \multicolumn{2}{c}{ Active } \\
\cline { 2 - 5 } & Maximum $(\mathbf{m m})$ & Average $(\mathbf{m m})$ & Maximum $(\mathbf{m m})$ & Average $(\mathbf{m m})$ \\
\hline Case 1 & 61.5 & 39.9 & 12.9 & 8.9 \\
Case 2 & 123.1 & 79.8 & 25.8 & 17.8 \\
\hline
\end{tabular}

Table 4 Results of the acceleration of the sprung mass.

\begin{tabular}{ccccc}
\hline & \multicolumn{3}{c}{ Passive } & Active \\
\cline { 2 - 5 } & Maximum $\left(\mathrm{m} / \mathrm{s}^{2}\right)$ & Average $\left(\mathrm{m} / \mathrm{s}^{2}\right)$ & Maximum $\left(\mathrm{m} / \mathrm{s}^{2}\right)$ & Average $\left(\mathrm{m} / \mathrm{s}^{2}\right)$ \\
\hline Case 1 & 1.295 & 0.386 & 0.748 & 0.086 \\
Case 2 & 2.590 & 0.773 & 1.501 & 0.172 \\
\hline
\end{tabular}

The percentages of variation between active and passive suspension use are summarized in Table 5 .

Table 5 Compare two suspension systems.

\begin{tabular}{ccccc}
\hline & \multicolumn{2}{c}{ Displacement } & \multicolumn{2}{c}{ Acceleration } \\
\cline { 2 - 5 } & Maximum (\%) & Average (\%) & Maximum (\%) & Average (\%) \\
\hline Case 1 & 20.98 & 22.31 & 57.76 & 22.28 \\
Case 2 & 20.96 & 22.31 & 57.95 & 22.25 \\
\hline
\end{tabular}

\section{CONCLUSION}

Oscillation can directly affect the stability and comfort of the vehicle during travel. Many factors cause a vehicle's oscillation, among which roughness on the road surface is the main cause of this phenomenon. The conventional passive suspension system cannot meet the requirements set for the smoothness and comfort of the vehicle. Therefore, the active suspension system is proposed to replace the mechanical suspension system.

This research proposes to use a quarter dynamics model to simulate the vehicle's oscillation. Besides, the dynamics model of the hydraulic actuator is also shown. This model is linearized with very small errors. Because the vehicle is a complex oscillating system, the Sliding Mode Control algorithm is proposed. This is a very complex nonlinear control method. In this paper, the author has established the sliding control method based on a linearized hydraulic actuator. This is a completely novel and unique solution. It is completely different from other conventional control methods.

The simulation results showed the superiority of the Sliding Mode Control algorithm. According to these results, the displacement and acceleration of the sprung mass were greatly reduced if the vehicle used the active suspension system. Besides, the oscillation of the unsprung mass is almost unchanged from another situation. In both cases, the performance of the controller is high. The vehicle's stability and smoothness have been significantly improved.

This research has only been performed in a quarter dynamics model. At the same time, the results of the research are shown based on the simulation process. In the future, more complex models can also be proposed. Besides, the experimental process is necessary to accurately evaluate the efficiency of this nonlinear control algorithm.

Author contributions: Conceptualization, T A Nguyen; Introduction, N D Dang; Material and Method, D N Nguyen; Simulation, T A Nguyen; Conclusions, D N Nguyen; Writing, T A Nguyen and N D Dang

Editor: Marcílio Alves

\section{References}

Abid, H. J., Chen, J., and Nassar, A. A. (2015). Equivalent Air Spring Suspension Model for Quarter-Passive Model of Passenger Vehicles, International Scholarly Research Notices, 2015.

Aela, A. M. A., Kenne, J. P., and Mintsa H. A. (2020). Adaptive Neural Network and Nonlinear Electrohydraulic Active Suspension Control System. Journal of Vibration and Control. 
Aljarbouh, A. and Fayaz, M. (2020). Hybrid Modelling and Sliding Mode Control of Semi-Active Suspension Systems for Both Ride Comfort and Roadholding, Symmetry, 12(8).

Alves, U. N. L. T., et al. (2014). Sliding Mode Control for Active Suspension System with Data Acquisition Delay, Mathematical Problems in Engineering, 2014.

Anh, N. T. (2020). Control an Active Suspension System by Using PID and LQR Controller. International Journal of Mechanical and Production Engineering Research and Development, 10(3): 7003-12.

Bai, R. and Guo, D. (2018). Sliding-Mode Control of the Active Suspension System with the Dynamics of a Hydraulic Actuator, Complexity, 2018.

Bello, M. M., Shafie, A. A., and Khan, R. (2015). Active Vehicle Suspension Control Using Full State-Feedback Controller, Advanced Materials Research, 1115:440-45.

Chai, L. and Sun, T. (2010). The Design of LQG Controller for Active Suspension Based on Analytic Hierarchy Process, Mathematical Problems in Engineering, 2020.

Chen, S., Cai, Y., Wang, J., and Yao, M. (2018). A Novel LQG Controller of Active Suspension System for Vehicle Roll Safety, International Journal of Control, Automation and Systems, 16:2203-13.

Eskandary, P. K., Khajepour, A., Wong, A., and Ansari, M. (2016). Analysis and Optimization of Air Suspension System with Independent Height and Stiffness Tuning, International Journal of Automotive Technology, 7(5):807-16.

Fu, Z. J., Li, B., Ning, X. B., and Xie, W. D. (2017). Online Adaptive Optimal Control of Vehicle Active Suspension Systems Using Single-Network Approximate Dynamic Programming, Mathematical Problems in Engineering, 2017.

Gomonwattanapanich O., et al. (2020). Vibration Control of Vehicle by Active Suspension with LQG Algorithm, International Journal of Automotive and Mechanical Engineering, 17(2):8011-18.

Gudarzi, M. and Oveisi, A. (2014). Robust Control for Ride Comfort Improvement of an Active Suspension System Considering Uncertain Driver's Biodynamics, Journal of Low Frequency Noise, Vibration and Active Control, 33(3):317-339.

Haddar, M., et al. (2019). Intelligent PD Controller Design for Active Suspension System Based on Robust Model-free Control Strategy, Journal of Mechanical Engineering Science, 233(14):4863-80.

Heidari, M. and Homaei, H. (2013). Design a PID Controller for Suspension System by Back Propagation Neural Network, Journal of Engineering, 2013.

Huang, Y., et al. (2015). Adaptive Control of Nonlinear Uncertain Active Suspension Systems with Prescribed Performance, ISA Transactions, 54:145-55.

Huang, Y., et al. (2018). Robust Adaptive Control for Vehicle Active Suspension Systems with Uncertain Dynamics. Transactions of the Institute of Measurement and Control, 40(4):1237-49.

Jiregna, I. and Sirata, G. (2020). A Review of the Vehicle Suspension System, Journal of Mechanical and Energy Engineering, 4(44):109-14.

Jugulkar, L. M., Singh, S., and Sawant, S. M. (2016). Analysis of Suspension with Variable Stiffness and Variable Damping Force for Automobile Applications, Advances in Mechanical Engineering, 8(5):1-19.

Kaleemullah, M., Faris, W., and Ghazaly, N. M. (2019). Analysis of Active Suspension Control Policies for Vehicle Using Robust Controllers, International Journal of Advanced Science and Technology, 28(16):836-55.

Li, W., Du, H., Ning, D., and Li, W. (2019). Robust Adaptive Sliding Mode PI Control for Active Vehicle Seat Suspension Systems, 2019 Chinese Control and Decision Conference, 5403-08.

Lin, B., Su, X., and Li, X. (2019). Fuzzy Sliding Mode Control for Active Suspension System with Proportional Differential Sliding Mode Observer, Asian Journal of Control, 21(1):1-13.

Liu, Y. J., et al. (2019). Adaptive Neural Network Control for Active Suspension Systems with Time-Varying Vertical Displacement and Speed Constraints, IEEE Transactions on Industrial Electronics, 66(12):9458-66.

Maurya, V. K. and Bhangal, N. S. (2018). Optimal Control of Vehicle Active Suspension System, Journal of Automation and Control Engineering, 6(1):22-6.

Mrazgua, J., Chaibi, R., Tissir, E. H., and Ouahi, M. (2021). Static Output Feedback Stabilization of T-S Fuzzy Active Suspension Systems. Journal of Terramechanics, 97:19-27.

Munawwarah, S. and Yakub, F. (2021). Control Analysis of Vehicle Ride Comfort Through Integrated Control Devices on the Quarter and Half Car Active Suspension Systems, Journal of Automobile Engineering, 235(5):1256-68. 
$\mathrm{Na}$, J., et al. (2020). Adaptive Finite-Time Fuzzy Control of Nonlinear Active Suspension Systems with Input Delay, IEEE Transactions on Cybernetics, 50(6):2639-50.

Nagarkar, M. P., Vikhe, G. J., Borole, K. R., and Nandedkar, V. M. (2011). Active Control of Quarter-Car Suspension System Using Linear Quadratic Regulator, International Journal of Automotive and Mechanical Engineering; 3(1):364-72.

Nguyen, T. A. (2021a). Advance the Stability of the Vehicle by Using the Pneumatic Suspension System Integrated with the Hydraulic Actuator, Latin American Journal of Solids and Structures, 18(7).

Nguyen, T. A. (2021b). Improving the Comfort of the Vehicle Based on Using the Active Suspension System Controlled by the Double-Integrated Controller, Shock and Vibration, 2021.

Nguyen, T. A. (2021c). Study on the Sliding Mode Control Method for the Active Suspension System. International Journal of Applied Science and Engineering, 18(5).

Palanisamy, S. and Karuppan, S. (2016). Fuzzy Control of Active Suspension System. Journal of Vibroengineering, 18(5):3197-204.

Pang, H., Chen, Y., Chen, J. N., and Liu, X. (2017). Design of LQG Controller for Active Suspension without Considering Road Input Signals, Shock and Vibration, 2017.

Rizvi S. M. H., et al. (2018). $H_{\infty}$ Control of 8 Degrees of Freedom Vehicle Active Suspension System, Journal of King Saud University - Engineering Sciences, 30(2):161-69.

Rodriguez-Guevara, D., et al. (2021). Active Suspension Control Using an MPC-LQR-LPV Controller with Attraction Sets and Quadratic Stability Conditions, Mathematics, 9(20).

Sam, Y. M. and Osman, J. H. S. (2006). Sliding Mode Control of a Hydraulically Actuated Active Suspension, Jurnal Teknologi, 44:37-48.

Sam, Y. M., Hudha, K., and Osman, J. H. S. (2007). Proportional-Integral Sliding Mode Control of a Hydraulically Actuated Active Suspension System: Force Tracking and Disturbance Rejection Control on Non-linear Quarter Car Model, International Journal of Vehicle Systems Modelling and Testing, 2(4):391-410.

Singh, N., Chhabra, H., and Bhangal, K. (2016). Robust Control of Vehicle Active Suspension System, International Journal of Control and Automation, 9(4):149-60.

Soleymani, M., Montazeri-Gh, M., and Amiryan, R. (2012). Adaptive Fuzzy Controller for Vehicle Active Suspension System Based on Traffic Conditions, Scientia Iranica, 19(3):443-53.

Sun, J. and Zhao, K. (2020). Adaptive Neural Network Sliding Mode Control for Active Suspension Systems with Electrohydraulic Actuator Dynamics, International Journal of Advanced Robotic Systems.

Sun, W., Zhao, Z., and Gao, H. (2013). Saturated Adaptive Robust Control for Active Suspension Systems, IEEE Transactions on Industrial Electronics, 60(9):3889-96.

Talib, M. H. A. and Darus, I. Z. M. (2013). Self-Tuning PID Controller for Active Suspension System with Hydraulic Actuator, 2013 IEEE Symposium on Computers \& Informatics: 86-91.

Talib, M. H. A., et al. (2021). Vibration Control of Semi-Active Suspension System Using PID Controller with Advanced Firefly Algorithm and Particle Swarm Optimization. Journal of Ambient Intelligence and Humanized Computing, 12:1119-37.

Tian, M. and Nguyen, V. (2020). Control Performance of Suspension System of Cars with PID Control Based on 3D Dynamic Model, Journal of Mechanical Engineering, Automation and Control Systems, 1(1):1-10.

Wang, D., Zhao, D., Gong, M., and Yang, B. (2018). Research on Robust Model Predictive Control for Electro-Hydraulic Servo Active Suspension Systems, IEEE Access, 6:3231-40.

Wei, S. and Su, X. (2020). Sliding Mode Control Design for Active Suspension Systems Using Quantum Particle Swarm Optimisation, International Journal of Vehicle Design, 81(1/2):93-114.

Wu, H., et al. (2020). Robust Control for Active Suspension of Hub-Driven Electric Vehicles Subject to in-Wheel Motor Magnetic Force Oscillation, Applied Sciences 10(11).

Xia, R. X., et al. (2015). Linear-Quadratic-Gaussian Controller for Truck Active Suspension Based on Cargo Integrity, Advances in Mechanical Engineering, 7(12):1-9.

Xiao, P., Gao, H., Shi, P., and Niu, L. (2018). Research on Air Suspension with Novel Dampers Based on Glowworm Swarm Optimization Proportional-Integral-Derivative Algorithm, Advances in Mechanical Engineering, 10(8):1-19.

Zhao, F., et al. (2015). Adaptive Neural-Sliding Mode Control of Active Suspension System for Camera Stabilization, Shock and Vibration, 2015. 